\title{
Results from a U.S. Dog Owner Survey on the Treatment Satisfaction and Preference for Fluralaner against Flea and Tick Infestations
}

\author{
Robert P Lavan ${ }^{1 *}$, Robert Armstrong ${ }^{2}$, Dorothy Normile ${ }^{3}$, Dongmu Zhang ${ }^{4}$ and Kaan Tunceli ${ }^{4}$ \\ ${ }^{1}$ Outcomes Research, Animal Health Center for Observational and Real-World Evidence, Merck \& Co. Inc., Kenilworth, NJ 07033, USA \\ ${ }^{2}$ Animal Health Global Marketing, Merck \& Co. Inc., Madison, NJ 07940, USA \\ ${ }^{3}$ Animal Health Technical Services, Merck \& Co. Inc., Madison, NJ 07940, USA \\ ${ }^{4}$ Center for Observational and Real-World Evidence, Merck \& Co. Inc., Kenilworth, NJ 07033, USA
}

\begin{abstract}
Background: Fluralaner is a potent acaricide and insecticide effective against flea and tick (F/T) infestations on dogs and cats. Fluralaner for dogs can be administered orally as a flavored chew with up to a 12-week redosing interval, about three-fold less frequently than monthly F/T medications. This study surveyed dog owners who currently administer fluralaner to their dogs to determine their level of satisfaction with the product and its perceived benefits compared to monthly medications, including the potential for on-time administration compliance.
\end{abstract}

Methods: In the period April to June 2016, dog-owner clients from 25 veterinary practices in 16 U.S. states completed a 10-item survey questionnaire $(n=559)$ that asked respondents about their experience with fluralaner and monthly medications. In multivariate analyses, predictors of treatment satisfaction and predictors of preference with fluralaner have been estimated by an ordered logistic regression and a logistic regression, respectively.

Results: Seventy-three percent of survey respondents had used monthly F/T medications prior to fluralaner. Respondents identified convenience (74\%), the 12-week dosing interval $(69 \%)$, and less-frequent dosing $(68 \%)$ as the three most important benefits of using fluralaner. Sixty six percent were very satisfied and $30 \%$ were satisfied with fluralaner and $89 \%$ preferred fluralaner versus monthly $\mathrm{F} / \mathrm{T}$ medications. Pet owners who used monthly $\mathrm{F} / \mathrm{T}$ products, $65 \%$ thought that they were more likely to give the next fluralaner dose on time compared to doses of monthly $\mathrm{F} / \mathrm{T}$ products, and $88 \%$ said that giving repeat doses of fluralaner was more convenient than giving monthly F/T products. In multivariable models, "12 weeks dosing/convenience" and "female gender" were positively associated with treatment satisfaction and preference with fluralaner $(p<0.05)$.

Conclusions: Overall satisfaction with fluralaner and preference for fluralaner compared to monthly $F / T$ medications were high. The most significant factor predicting satisfaction and preference was perceived benefit with 12 weeks dosing or convenience.

Keywords: Bravecto; Canine; Compliance; Fleas; Ticks; Ectoparasite; Ectoparasiticide; Acaricide; Insecticide; Isoxazoline

\section{Background}

Canine flea-and-tick (F/T) medications in the United States are generally owner-administered and available for purchase from veterinarians or other retail locations. At this time, the isoxazoline class of $\mathrm{F} / \mathrm{T}$ medications, including afoxolaner, fluralaner, and sarolaner, are only available by prescription through veterinarians. The high environmental and host prevalence and persistence of the target parasites, their role in flea allergy dermatitis (FAD) and vector-borne diseases, and the routine movement of infested dogs into previously non-endemic urban and suburban areas are factors that support the importance of ectoparasite control [1-3]. There is a wide assortment of approved oral and topical canine $\mathrm{F} / \mathrm{T}$ medications with various modes of action and in various combinations, including some with insect growth regulator activity and some with canine heartworm (HW) indications. Fluralaner is a recently approved active ingredient in the isoxazoline class available in oral chewable formulation (Fluralaner, Merck Animal Health, Madison, NJ, USA) for dogs and a topical formulation for dogs and cats $[4,5]$. The fluralaner re-treatment interval is three times longer than the re-treatment interval for other flea/tick products that are dosed every 4 weeks. Fluralaner offers a new approach to canine F/T control, based on rapid and persistent acaricidal and insecticidal activity [6-8].

Clinical trials have demonstrated the ectoparasitic efficacy of fluralaner as a gamma-aminobutyric acid (GABA) and glutamategated chloride channel inhibitor with potent selectivity for arthropod neuron receptors, resulting in excess neuronal stimulation and rapid insect death after feeding exposure $[9,10]$. After oral ingestion by the dog, fluralaner is rapidly absorbed and widely distributed, with a long half-life of 12-15 days. Fluralaner undergoes negligible host metabolism and is primarily excreted unchanged in the feces [11].

Fluralaner safety in dogs has been extensively evaluated, including dose tolerance studies with administration at up to five times the maximum label dose $[4,12,13]$. Fluralaner is unusual in the isoxazoline class of ectoparasiticides in that it has sustained activity that allows 12 week re-dosing [4] while the other isoxazolines are re-dosed at 4 week intervals.

Poor compliance is a leading cause of treatment failure in $\mathrm{F} / \mathrm{T}$ and HW control as has been shown for other therapeutic regimens

*Corresponding author: Robert P Lavan, Outcomes Research, Animal Health Center for Observational and Real-World Evidence, Merck \& Co. Inc., Kenilworth, NJ 07033, USA, Tel: (908) 740-4000; E-mail: robert.lavan@merck.com

Received April 05, 2017; Accepted April 19, 2017; Published April 21, 2017

Citation: Lavan RP, Armstrong R, Normile D, Zhang D, Tunceli K (2017) Results from a U.S. Dog Owner Survey on the Treatment Satisfaction and Preference for Fluralaner against Flea and Tick Infestations. J Vet Sci Technol 8: 439. doi 10.4172/2157-7579.1000439

Copyright: $\odot 2017$ Lavan RP, et al. This is an open-access article distributed unde the terms of the Creative Commons Attribution License, which permits unrestricted use, distribution, and reproduction in any medium, provided the original author and source are credited. 
in veterinary and human medicine [14-17]. Compliance shortfalls are primarily influenced by the complexity and convenience of the therapeutic protocol. For example, Canadian investigators found that compliance declined significantly as the number of antimicrobial doses administered by dog owners increased [18]. Dog owners who were asked to give oral antimicrobial drugs once or twice daily to their dogs were nine times more likely to be fully compliant compared with owners who were asked to treat three-times daily. Similarly, in a European study, only $44 \%$ of dog owners $(n=95)$ were fully compliant with a 10-day course of oral anti-infective therapy [19]. These results show that extended, multi-dose regimens are inherently more likely to be associated with missed doses and lack of on-time dosing. Simpler, less frequent dosing is directly related to better compliance across all therapeutic classes [20].

Monthly (or every 4 weeks) administration of oral or topical canine $\mathrm{F} / \mathrm{T}$ and $\mathrm{HW}$ medications is common in companion animal veterinary practice and has become a standard dosing schedule. However, even a monthly dosing frequency does not lead to a high level of compliance in using these parasiticides, which are recommended for year round administration by animal health groups $[21,22]$. Recent data for canine patients $(n=1,271)$ presented at a U.S. veterinary teaching hospital found that $74 \%$ were being treated with $\mathrm{F} / \mathrm{T}$ preventive products and only $61 \%$ of those ( $45 \%$ of total population) received the products year around [16]. The authors considered this level of compliance for veterinary referral center patients to be much higher than that for the general dog population and suggested that improvement in owner compliance with treatment recommendations could provide better F/T control.

Using a cohort of dog owners who have recent experience giving fluralaner to their dogs, the objectives of this study were:

To use a survey to assess treatment satisfaction and preference in dog owners that have given at least two doses of fluralaner and may have also given shorter acting (monthly) ectoparasiticides to their dogs.

$>$ Describe predictors of satisfaction and preference.

$>$ Assess dog owner difficulty adapting to 12 week dosing.

The survey included pet owners from geographically diverse regions of the U.S., including F/T endemic areas of the Eastern, Central, Southeastern and Western United States. Veterinarians in each of these clinics were also asked to provide the number of months that they routinely recommend flea/tick protection for their canine patients.

\section{Methods}

\section{Survey population}

Twenty-six veterinarians from 24 practices located in 16 states in various regions of the U.S. participated in the study (Table 1) during the period April to June, 2016. The veterinarians averaged 17.4 years in practice and included 11 male and 15 female practitioners. Each participating practice recruited dog owners whose pets were currently being treated with fluralaner and who had purchased $\geq 2$ doses, and invited them to complete the survey questionnaire. Practices that dispensed various $\mathrm{F} / \mathrm{T}$ products in addition to fluralaner were included in the study. Dog owners had various experiences with the prior use of flea and tick products although all were currently giving fluralaner to their dogs.

559 dog owners completed survey questionnaires after providing information on their age (10-year age block), gender, years as a caretaker for their dog as well as their experience with fleas and ticks. Information for each dog included the dog's age, gender and neutering status, bodyweight, health assessment, summary of outdoor activities and time spent outdoors (Table 2).

\section{Survey instrument}

The dog owner questionnaire consisted of 10 questions related to utilization of F/T products, as shown in Table 3 . The survey was developed and pretested in an iterative review process. Several survey questions invited the responder to make free-text written comments, thus providing an opportunity for more detailed, specific or alternate responses. The survey was designed to be completed in $<5$ minutes and was administered while they were in the veterinary clinic or by telephone if they had recently been in the clinic. The first survey question allowed the identification of a dog owner cohort subset who had used prior F/T products in addition to fluralaner. This cohort was particularly valuable in providing responses to questions 7-10 which compared 12 week dosing (fluralaner) to 4 week dosing (other monthly F/T products).

\section{Data analysis}

Responses to each survey question were examined for differences by pet owner gender, age, years as dog caretaker, geographic region, dog outdoor time and outdoor activities as well as the pet owners' F/T experience. Descriptive statistics (number/percentage or mean/SD) were used to describe responses for each survey question. All statistical analyses were performed using SAS version 9.3 (SAS Institute, Cary, NC). Significances of differences between variables were determined by analysis of variance (ANOVA) or Chi-Square test with values of $\mathrm{P}<0.05$ considered statistically significant. Ordered logistic regression was

\begin{tabular}{|c|c|c|c|c|}
\hline U.S. Region & $\begin{array}{c}\text { Practices } \\
(\#)\end{array}$ & $\begin{array}{c}\text { Surveys } \\
(\#)\end{array}$ & $\begin{array}{c}\text { Participating } \\
\text { veterinarians (\#) }\end{array}$ & $\begin{array}{c}\text { Mean years } \\
\text { in practice }\end{array}$ \\
\hline Northeast (NY, OH, PA) & 4 & 92 & $\mathbf{5}$ & $\mathbf{6 . 0} \pm \mathbf{4 . 2}$ \\
\hline $\begin{array}{c}\text { Central (AR, IL, IN, KS, } \\
\text { KY, MO) }\end{array}$ & 8 & 192 & $\mathbf{8}$ & $\mathbf{1 6 . 0 \pm 8 . 6}$ \\
\hline South (AL, FL, GA, TX) & 9 & 191 & $\mathbf{9}$ & $\mathbf{2 2 . 8} \pm \mathbf{1 0 . 6}$ \\
\hline West (AZ, CA, HI) & 3 & 84 & $\mathbf{4}$ & $\mathbf{1 9 . 5} \pm \mathbf{1 . 4}$ \\
\hline Total & 24 & 559 & $\mathbf{2 6}$ & $\mathbf{1 7 . 4 \pm 1 0 . 7}$ \\
\hline
\end{tabular}

Table 1: Location of veterinary practices participating in the dog-owner treatment satisfaction survey.

\begin{tabular}{|l|c|}
\hline Population characteristics & Description \\
\hline Owner Demographic Factors & \\
\hline Gender & $390 \mathrm{f}, 140 \mathrm{~m}$ \\
Age 10-49 years & $232(41.5 \%)$ \\
$50-69$ years & $223(39.9 \%)$ \\
$70+$ years & $41(7.3 \%)$ \\
Years as Dog's Caretaker (Mean(SD)) & $5.5(3.6)$ \\
\hline Canine Demographic Factors & \\
\hline Gender & $288 \mathrm{~m}, 270 \mathrm{f}$ \\
Neutered or spayed & $89 \%$ \\
Age (Mean) & 6.1 years \\
Owner reports good or excellent health & $92 \%$ \\
\hline Flea-and-tick exposure risk factors & \\
\hline Ave. time spent outdoors & 4.2 hours/day \\
Swims & 1 in 4 \\
Goes to dog park & 1 in 3 \\
\hline Has access to woods & 1 in 2.5 \\
Walks off leash or has access to high or uncut grass & 1 in 2 \\
Owner reported seeing fleas on their dogs & $41 \%$ \\
Owner reported seeing ticks on their dogs & $31 \%$ \\
Owner reported seeing ticks on family members & $11 \%$ \\
\hline
\end{tabular}

Table 2: Demographic profile and ectoparasite exposure risk factors for participating owners and their dogs. 
Citation: Lavan RP, Armstrong R, Normile D, Zhang D, Tunceli K (2017) Results from a U.S. Dog Owner Survey on the Treatment Satisfaction and Preference for Fluralaner against Flea and Tick Infestations. J Vet Sci Technol 8: 439. doi: 10.4172/2157-7579.1000439

Survey question and response options

\section{Response rate}

Q1. Have you used flea-tick control products other than Fluralaner in the past?

\begin{tabular}{|c|c}
\hline Yes & $\%(\mathbf{n})$ dog owners $(\mathbf{n = 5 5 3}$ \\
\hline No & $73(406)$ \\
\hline Don't know & $20(108)$ \\
\hline
\end{tabular}

Q2. What is your level of satisfaction with Fluralaner?

$\%$ (n) dog owners ( $n=544$ respondents)

\begin{tabular}{|c|c} 
Very satisfied & $66(361)$ \\
Satisfied & $30(161)$ \\
\hline Neutral & $3(19)$ \\
\hline fied or very unsatisfied & $1(3)$
\end{tabular}

Unsatisfied or very unsatisfied
\[ \text { Q3. Which Fluralaner benefits are important to you (indicate any that apply)? } \]

$\%$ (n) dog owners ( $n=559$ respondents)

\begin{tabular}{|c|c|}
\hline Convenience & \% $(\mathbf{n})$ dog owners $(\mathbf{n}=559$ \\
\hline 12-week dosing interval & $67(305)$ \\
\hline Dosing less often than before & $67(373)$ \\
\hline Quickly kills F/Ts & $45(253)$ \\
\hline Easier dosing creates less stress & $45(251)$ \\
\hline Palatability & $43(240)$
\end{tabular}

Q4. If you had to select one benefit, which one is most important reason to use Fluralaner?

12-week dosing interval $23(131)$

Convenience $19(106)$

Dosing less often $\quad 18(102)$

Quickly kills F/Ts $\quad 17(96)$

\begin{tabular}{l|l} 
Easier dosing creates less stress & $9(49)$
\end{tabular}

\begin{tabular}{l|l} 
Palatability & $4 \%(25)$
\end{tabular}

Q5. Does using a product that lasts 12 weeks have an advantage over F/T products that require monthly dosing (indicate all that apply)?

Dogs is less likely to get fleas

Owner is less likely to forget a dose

Owner can give treatment less often

Dog is less likely to get ticks

Dog is less likely to bring fleas-ticks into house

Dog is more likely to be protected when it matters

Dog is less likely to itch
$\%$ (n) of all dog owners ( $n=559$ respondents)

$54(302)$
$52(288)$
$47(263)$
$44(244)$
$41(230)$
$35(193)$
$30(169)$

$\%$ (n) of dog owners who have used Fluralaner and other $F / T$ products ( $n=406$ respondents)

$$
\begin{aligned}
& 54(221) \\
& 53(215) \\
& 46(188) \\
& 44(177) \\
& 42(171) \\
& 34(139) \\
& 30(123)
\end{aligned}
$$

\begin{tabular}{|c|c|}
\hline & $\%(n)$ dog owners ( $n=509$ respondents) \\
\hline Mostly on time & $75(380)$ \\
\hline Delayed by a few days & $17(86)$ \\
\hline Delayed by weeks & $6(31)$ \\
\hline Delayed by months & $2(12)$ \\
\hline \multicolumn{2}{|c|}{ Q7. Are you more likely to give the next Fluralaner dose on time compared to monthly $F / T$ products? } \\
\hline & $\%(n)$ dog owners who have used Fluralaner and other $F / T$ products ( $n=381$ respondents) \\
\hline Yes & $65(246)$ \\
\hline No & $4(15)$ \\
\hline About the same & $27(102)$ \\
\hline Don't know & $4(18)$ \\
\hline \multicolumn{2}{|c|}{ Q8. With Fluralaner, my dog has (fewer/same/more) months of F/T protection in a year. } \\
\hline & $\%$ (n) dog owners who have used Fluralaner and other $F / T$ products ( $n=335$ respondents) \\
\hline Fewer & $8(26)$ \\
\hline Same & $56(189)$ \\
\hline More & $36(120)$ \\
\hline \multicolumn{2}{|c|}{ Q9. Is it (more/equally/less) convenient to give repeat doses of Fluralaner than repeat doses of a monthly F/T product? } \\
\hline & $\%(n)$ dog owners who have used Fluralaner and other $F / T$ products ( $n=382$ respondents) \\
\hline More & $89(339)$ \\
\hline
\end{tabular}

Q6. When you use Fluralaner during a given F/T season, is there a delay beyond the recommended 12-weeks in giving the next dose? I give the next dose: 


\begin{tabular}{|c|c|c|}
\hline \multicolumn{2}{|c|}{ Equally } & $10(39)$ \\
\hline Less & 1(4) \\
\hline & Q10. Do you prefer Fluralaner to other F/T product you have used? \\
\hline & $\%$ (n) dog owners who have used Fluralaner and other F/T products ( $\mathbf{n}=\mathbf{3 8 3}$ respondents) \\
\hline Yes & $89(341)$ \\
\hline No & $1(5)$ \\
\hline About the same & $10(37)$ \\
\hline
\end{tabular}

Table 3: Dog owner responses to a flea-and-tick (F/T) treatment survey.

used to understand the factors associated with pet owners' satisfaction of Bravecto. Satisfaction was measured as three levels: very satisfied, satisfied, and none-satisfied. Ordered logistic regression is an ordinal regression model-that is, a regression model for ordinal dependent variables. It is an extension of the logistic regression model that applies to dichotomous dependent variables, allowing for more than two ordered response categories. Binomial logistic regression was used to explore the factors associated with pet owners' preference of Bravecto. Preference was measured as two levels: Preferred vs. Non-preferred. The examined factors include pet owners' age, gender, number of years as a caregiver of the dog, seeing flee/tick on the dog, seeing tick on family members, identifying ' 12 weeks of dosing' or 'convenience' as one of Bravecto's benefits, dogs' age, weight, number of hours spending outside. Significant differences were noted with values of $\mathrm{P}<0.05$.

\section{Results}

On average, veterinarians from 24 practices in 16 states reported that they recommended 12 months of flea and tick protection for their canine patients. Only two veterinarians had different opinions related to year-round coverage. One veterinarian in the Southern region recommended 9 months of tick control and one veterinarian in the Northeast region recommended 8 months of flea control.

Five hundred fifty nine pet owners submitted completed surveys although not all pet owners chose to answer every question. Survey respondents (Table 2) were most often female $(70 \%, \mathrm{n}=390)$ and in the 50-59 year age block and as seen from survey question 1 , frequently were familiar with $\mathrm{F} / \mathrm{T}$ medications other than Fluralaner $(73 \%$, 406/553).

The demographic profile of the canine patients is shown in Table 2 . The canine population was evenly divided between male and female dogs, the great majority of which were neutered or spayed and in good or excellent health. The dogs had a relatively high risk of $\mathrm{F} / \mathrm{T}$ exposure as indicated by access to various outdoor settings, an average of 4.2 hours/ day spent outdoors, and a high percentage of on-animal ectoparasite sightings. Owners reported seeing fleas or ticks on their dogs in $41 \%$ and $31 \%$ of the cases, respectively, and $11 \%$ reported seeing ticks on family members.

A summary of responses are shown for the 10 survey questions (Table 3). While all participating pet owners were encouraged to answer all questions, the responses for a subset of pet owners who had used other flea/tick products were used to address questions 5 and 7 through 10, particularly because these questions compared current fluralaner use with prior use of $\mathrm{F} / \mathrm{T}$ products that were dosed monthly.

The majority of dog-owners (96\%) were either "satisfied" (30\%) or "very satisfied" (66\%) with their fluralaner experience. In the ordered logistic regression analysis of predictors of satisfaction (Table 4), female pet owners were significantly more likely to be "satisfied" compared to male pet owners $(\mathrm{P}=0.004)$, pet owners over 70 years of age were less likely to be satisfied ( $\mathrm{P}=0.036)$ compared to younger pet owners and pet owners who selected "12 week dosing" or "convenience" as preferred features of fluralaner were significantly more likely $(\mathrm{P}=0.001)$ to be satisfied compared to pet owners who has not selected " 12 week dosing" or "convenience" after adjusting other variables in the model.

Questions 3 and 4 asked responders to identify any benefits they associated with using fluralaner. A majority of participating dog owners most often selected convenience (72\%), 12-week dosing (67\%), and dosing less often $(67 \%)$ as important perceived benefits of fluralaner. Less frequently, respondents selected choices related to flea efficacy and their responsibility for giving the next dose on time. When asked about advantages associated with a longer lasting $\mathrm{F} / \mathrm{T}$ product (question 5), dog owners most frequently chose responses related to "dog is less likely to get fleas" and "owner is less likely to forget a dose". Several questions (Questions 6-9) asked about the pet owner experience with 12 week dosing. Three quarters (75\%) of dog owners stated that they thought that they gave fluralaner on time (question 6) and $17 \%$ reported that they administered the next dose with a few days delay suggesting that most pet owners thought that fluralaner was given in a more-or-less timely fashion ( $92 \%$ combined).

Questions 7-10 responses were drawn from dog owners who had used monthly F/T products in the past. Most respondents (65\%) thought that they were more likely to give the next fluralaner dose on time compared to follow-on dose of monthly flea and tick medications (question 7). An smaller proportion (27\%) said that they would be as just as likely to administer a dose of fluralaner on time as they would a dose of a monthly $\mathrm{F} / \mathrm{T}$ medications. In terms of perceived months of $\mathrm{F} / \mathrm{T}$ protection that their dogs received (question 8 ), approximately half of dog owners reported that their dogs got the same number of months of coverage, while approximately $1 / 3$ of respondents thought that their dogs got more months of coverage with fluralaner. Most respondents $(89 \%)$ found fluralaner to be more convenient (question 9) and preferred fluralaner compared to monthly F/T medications (question 10).

In the binary logistic regression analysis of predictors of preference (Table 5), female pet owners were significantly more likely to prefer fluralaner over monthly $\mathrm{F} / \mathrm{T}$ products compared to male pet owners $(\mathrm{P}=0.047)$ and pet owners who selected "12 week dosing" or "convenience" as preferred features of fluralaner were significantly more likely $(\mathrm{P}=0.002)$ to prefer fluralaner compared to monthly $\mathrm{F} / \mathrm{T}$ products after adjusting the other variables.

\section{Discussion}

Most veterinarians in this study recommended 12 months of flea and tick coverage for their canine patients. Ectoparasite exposure can be seasonal in the United States, depending on the region and harshness of the winter. In recent years, flea and tick control has been more important year-round because of the mildness and warming of 
Citation: Lavan RP, Armstrong R, Normile D, Zhang D, Tunceli K (2017) Results from a U.S. Dog Owner Survey on the Treatment Satisfaction and Preference for Fluralaner against Flea and Tick Infestations. J Vet Sci Technol 8: 439. doi: 10.4172/2157-7579.1000439

Page 5 of 6

\begin{tabular}{|c|c|c|c|c|}
\hline Variable & Value & Odds Ratio & $95 \% \mathrm{Cl}$ & p-value \\
\hline \multirow[t]{2}{*}{ Owner gender } & Male & Reference & & \\
\hline & Female & 1.930 & $(1.241,3.002)$ & 0.004 \\
\hline \multirow[t]{3}{*}{ Owner age } & $10-49$ & Reference & & \\
\hline & $50-69$ & 0.843 & $(0.540,1.316)$ & 0.453 \\
\hline & $70+$ & 0.454 & $(0.218,0.948)$ & 0.036 \\
\hline \multirow[t]{2}{*}{ Flea on the dog } & No & Reference & & \\
\hline & Yes & 0.779 & $(0.505,1.202)$ & 0.259 \\
\hline \multirow[t]{2}{*}{ Tick on the dog } & No & Reference & & \\
\hline & Yes & 0.934 & $(0.582,1.497)$ & 0.775 \\
\hline \multirow[t]{2}{*}{ Tick on family } & No & Reference & & \\
\hline & Yes & 1.232 & $(0.625,2.428)$ & 0.547 \\
\hline Owner yrs of care & & 0.933 & $(0.821,1.059)$ & 0.283 \\
\hline Dog age & & 1.030 & $(0.909,1.168)$ & 0.643 \\
\hline Dog weight & & 0.997 & $(0.991,1.004)$ & 0.459 \\
\hline Dog out door hours & & 1.013 & $(0.969,1.059)$ & 0.575 \\
\hline \multirow[t]{2}{*}{12 Week dosing or convenience } & No & Reference & & \\
\hline & Yes & 2.754 & $(1.533,4.947)$ & 0.001 \\
\hline
\end{tabular}

*Treatment Satisfaction Categories: Very Satisfied, Satisfied, and Non-satisfied. Interpretation of odds ratios in the ordered logistic model: In the example with gender variable, 1.930 is the proportional odds ratio of comparing females to males on satisfaction adjusted with all other variables in the model. For females, the odds of very satisfied versus the combined satisfied and non-satisfied are 1.930 times higher than for males. Likewise, the odds of the combined categories of very satisfied and satisfied versus non-satisfied is also 1.930 times higher for females compared to males.

$\mathrm{N}=450$ after excluding the pet owners with missing values for any of the variables

Table 4: Predictors of Pet Owners Satisfaction*.

\begin{tabular}{|c|c|c|c|c|}
\hline Variable & Value & Odds Ratio & $95 \% \mathrm{Cl}$ & p-value \\
\hline \multirow{2}{*}{ Owner gender } & Male & Reference & & \\
\hline & Female & 1.977 & $(1.009,3.876)$ & 0.047 \\
\hline \multirow[t]{3}{*}{ Owner age } & $10-49$ & Reference & & \\
\hline & $50-69$ & 0.704 & $(0.348,1.425)$ & 0.330 \\
\hline & $70+$ & 0.604 & $(0.195,1.867)$ & 0.381 \\
\hline \multirow[t]{2}{*}{ Flea on the dog } & No & Reference & & \\
\hline & Yes & 1.514 & $(0.745,3.076)$ & 0.251 \\
\hline \multirow[t]{2}{*}{ Tick on the dog } & No & Reference & & \\
\hline & Yes & 1.598 & $(0.711,3.591)$ & 0.257 \\
\hline \multirow[t]{2}{*}{ Tick on family } & No & Reference & & \\
\hline & Yes & 0.584 & $(0.226,1.510)$ & 0.267 \\
\hline Owner yrs of care & & 0.846 & $(0.659,1.085)$ & 0.188 \\
\hline Dog age & & 1.125 & $(0.874,1.447)$ & 0.361 \\
\hline Dog weight & & 1.005 & $(0.994,1.016)$ & 0.389 \\
\hline Dog out door hours & & 1.013 & $(0.936,1.096)$ & 0.749 \\
\hline \multirow[t]{2}{*}{12 Week dosing or convenience } & No & Reference & & \\
\hline & Yes & 3.355 & $(1.564,7.198)$ & 0.002 \\
\hline $\begin{array}{l}\text { Preferred: } 1 \text {, Non-preferred: } 0 \\
\mathrm{~J}=450 \text { after excluding the pet owners }\end{array}$ & es for ar & & & \\
\hline
\end{tabular}

Table 5: Predictors of Pet Owners' Preference.

the winters. In regions like the Southern and Western United States, ectoparasites are routinely found outdoors all year long. The yearround need for ectoparasite control is reflected in the recommendation by the U.S. veterinarians.

The dogs in this study faced assorted risks of flea and tick exposure, including an average of 4.2 hours a day spent outdoors and access to wooded or grassy areas in $\geq 40 \%$ of the cases. Seventy three percent of pet owners in this study indicated that they had used flea and tick control products other than fluralaner in the past and thus were well suited to render an informed opinion on the relative perceived merits of $\mathrm{F} / \mathrm{T}$ medications.

The study participant pool was approximately $3 / 4$ female / $1 / 4$ male with the highest proportion of pet owners in the "middle aged" groups.
This may be more typical of the pet owner demographic that brings the dog to the clinic. The majority of pet owners were satisfied (30\%) or very satisfied $(60 \%)$ with their experience using fluralaner. It appears to be "12 week dosing" or "convenience" is an important perceived benefit for satisfaction with fluralaner. It is not clear why female pet owners were more likely to be satisfied compared to male pet owners. It is also not clear why older pet owners (70 years of age and older versus youngest age category " $<49$ years") were significantly less likely to be satisfied with fluralaner. Nevertheless, satisfaction was high across all age groups, regardless of the age of the pet owner or number of years that they had been the dog caretaker.

The three benefits of fluralaner that were most often selected were related to the longer dosing interval; convenience (72\%), 12 week 
Citation: Lavan RP, Armstrong R, Normile D, Zhang D, Tunceli K (2017) Results from a U.S. Dog Owner Survey on the Treatment Satisfaction and Preference for Fluralaner against Flea and Tick Infestations. J Vet Sci Technol 8: 439. doi: 10.4172/2157-7579.1000439

Page 6 of 6

dosing interval (67\%), and dosing less often (67\%). The responses of " 12 week dosing" was selected most often when dog owners were asked to select the single most important benefit of fluralaner. In a comparison to other F/T medications with monthly dosing, the majority of dog owners associated fluralaner with a reduced likelihood of getting fleas and a reduced likelihood of forgetting a dose. It is conceivable that pet owners might associate less forgotten doses with improved flea efficacy although there were no questions that specifically addressed this relationship. The next dose given "mostly on time" $(75 \%)$ or "delayed by a few days" (17\%) indicates that 12 -weeks administration of fluralaner is easy to achieve by dog owners.

In comparisons with monthly $\mathrm{F} / \mathrm{T}$ medications, most dog owners in our study appeared to favor fluralaner with regard to on time administration (65\%), convenience for repeat doses (89\%), and preference $(89 \%)$ although the majority believes $(56 \%)$ that dogs receive the same number of months of $\mathrm{F} / \mathrm{T}$ protection in a year. The dog owners in this study did not seem to have any problem adjusting to the longer dosing interval.

As suggested in the analyses examining predictors of preference, dog owners who perceive "12 week dosing or convenience" as an important benefit of fluralaner were more likely to prefer fluralaner over monthly $\mathrm{F} / \mathrm{T}$ medications. It is not clear why female pet owners were significantly more likely to state a preference for fluralaner over the monthly F/T products; a similar result found in the satisfaction analysis.

Medications with extended re-dosing intervals have been shown to improve patient compliance with veterinarian and physician recommendations when compared with medications that have shorter re-dosing intervals (15-17). Results from our study indicate that the 12 week re-treatment interval feature of fluralaner offering a longer approach to canine F/T control is associated with high satisfaction, convenience and preference scores in a group of dog owners that had currently been prescribed fluralaner for their dogs.

This study is based on the survey results of dog owners currently providing fluralaner to their own dogs. As such, it relies on pet owner opinion and is limited because it is a single-arm study design that did not directly compare fluralaner against specific monthly $\mathrm{F} / \mathrm{T}$ products. Future studies that examine satisfaction, preference, and compliance comparing 12-week dosing to other $\mathrm{F} / \mathrm{T}$ medications with monthly dosing are needed to fully evaluate the real-world experience of dog owners.

\section{Conclusion}

Overall satisfaction with fluralaner and preference for fluralaner compared to monthly F/T medications were high. The most significant factor predicting satisfaction and preference was the perceived benefit associated with 12 week dosing or convenience.

\section{Acknowledgments}

The authors thank Mark Dana of Scientific Communications Services, LLC for assistance in manuscript preparation.

\section{References}

1. Chomel B (2011) Tick-borne infections in dogs-an emerging infectious threat. Vet Parasitol 179: 294-301.

2. Shaw SE, Day MJ, Birtles RJ, Breitschwerdt EB (2001) Tick-borne infectious diseases of dogs. Trends Parasitol 17: 74-80.

3. Yancey CB, Hegarty BC, Qurollo BA, Levy MG, Birkenheuer AJ, et al. (2014) Regional seroreactivity and vector-borne disease co-exposures in dogs in the
United States from 2004-2010: utility of canine surveillance. Vector Borne Zoonotic Dis 14: 724-732.

4. Fluralaner Chewable Tablets Dogs. Freedom of Information Summary. Original New Animal Drug Application (NADA 141-426). May 15, 2014.

5. Williams H, Young DR, Qureshi T, Zoller H, Heckeroth AR (2014) Fluralaner, a novel isoxazoline, prevents flea (Ctenocephalides felis) reproduction in vitro and in a simulated home environment. Parasit Vectors 7: 275.

6. Pfister K, Armstrong R (2016) Systemically and cutaneously distributed ectoparasiticides: a review of the efficacy against ticks and fleas on dogs. Parasit Vectors 9: 436

7. Taenzler J, Wengenmayer C, Williams H, Fourie J, Zschiesche E, et al. (2014) Onset of activity of fluralaner (FLURALANER ${ }^{\mathrm{TM}}$ ) against Ctenocephalides felis on dogs. Parasit Vectors 7: 567.

8. Meadows C, Guerino F, Sun F (2014) A randomized, blinded, controlled USA field study to assess the use of fluralaner tablets in controlling canine flea infestations. Parasit Vectors 7: 375

9. Crosaz O, Chapelle E, Cochet-Faivre N, Ka D, Hubinois C, et al. (2016) Open field study on the efficacy of oral fluralaner for long-term control of flea allergy dermatitis in client-owned dogs in lle-de-France region. Parasit Vectors 9: 174

10. Kilp S, Ramirez D, Allan MJ, Roepke RK, Nuernberger MC (2014) Pharmacokinetics of fluralaner in dogs following a single oral or intravenous administration. Parasit Vectors 7: 85.

11. Dryden MW, Smith V, Bennett T, Math L, Kallman J, et al. (2015) Efficacy of fluralaner flavored chews (Fluralaner) administered to dogs against the adult cat flea, Ctenocephalides felis felis and egg production. Parasit Vectors 8: 364

12. Walther FM, Allan MJ, Roepke RK, Nuernberger MC (2014) Safety of fluralaner chewable tablets (Fluralaner), a novel systemic antiparasitic drug, in dogs after oral administration. Parasit Vectors 7: 87.

13. Walther FM, Paul AJ, Allan MJ, Roepke RK, Nuernberger MC (2014) Safety of fluralaner, a novel systemic antiparasitic drug, in MDR1(-/-) Collies after oral administration. Parasit Vectors 7: 86.

14. Rohdich N, Roepke RK, Zschiesche E (2014) A randomized, blinded, controlled and multi-centered field study comparing the efficacy and safety of Bravecto ${ }^{\mathrm{TM}}$ (fluralaner) against Frontline (fipronil) in flea- and tick-infested dogs. Parasit Vectors 7: 83

15. Beck S, Schreiber C, Schein E, Krücken J, Baldermann C, et al. (2014) Tick infestation and prophylaxis of dogs in northeastern Germany: a prospective study. Ticks Tick Borne Dis 5: 336-342.

16. Gates MC, Nolan TJ (2010) Factors influencing heartworm, flea, and tick preventative use in patients presented to a veterinary teaching hospital. Prev Vet Med 93: 193-200.

17. Van VI, Nautrup BP, Gasper SM (2011) Estimation of the clinical and economic consequences of non-compliance with antimicrobial treatment of canine skin infections. Prev Vet Med 99: 201-210.

18. Adams VJ, Campbell JR, Waldner CL, Dowling PM, Schmon CL (2005) Evaluation of client compliance with short-term administration of antimicrobials to dogs. J Am Vet Med Assoc 226: 567-574

19. Grave K, Tanem H (1999) Compliance with short-term oral antibacterial drug treatment in dogs. J Small Anim Pract 40: 158-162.

20. Claxton AJ, Cramer J, Pierce C (2001) A systematic review of the association between dose regimens and medication compliance. Clin Ther 23: 1296-1310.

21. Companion Animal Parasite Council: CAPC recommendations. Accessed Nov 22, 2016.

22. American Heartworm Society: Summary of the current canine guidelines for the prevention, diagnosis, and management of heartworm (Dirofilaria immitis) infection in dogs. Accessed Nov 22, 2016. 